\title{
Phase diagram of carbon dioxide revisited
}

\author{
K. F. Dziubek', D. Scelta ${ }^{1,2}$, M. Ende ${ }^{3}$, R. Miletich ${ }^{3}$, R. Bini ${ }^{1,2,4}$, M. Mezouar ${ }^{5}$, G. Garbarino ${ }^{5}$ \\ ${ }^{1}$ LENS, European Laboratory for Non-linear Spectroscopy, Via N. Carrara 1, I-50019 Sesto Fiorentino, Italy, \\ ${ }^{2}$ ICCOM-CNR, National Research Council of Italy, Via Madonna del Piano 10, I-50019 Sesto Fiorentino, Italy, \\ ${ }^{3}$ Institut für Mineralogie und Kristallographie, Universität Wien, Althanstrasse 14, A-1090 Wien, Austria, \\ ${ }^{4}$ Dipartimento di Chimica "Ugo Schiff" dell'Università degli Studi di Firenze, Via della Lastruccia 3, I-50019 Sesto Fiorentino, Italy, \\ ${ }^{5}$ European Synchrotron Radiation Facility, ESRF, 71 avenue des Martyrs, CS 40220, 38043 Grenoble Cedex 9, France

$$
\text { dziubek@lens.unifi.it }
$$

Carbon dioxide, $\mathrm{CO}_{2}$, is one of the most important compounds in nature and the second most abundant volatile in the Earth's interior. Its structure and properties at high pressures and temperatures pertaining to geoscience are crucial both to fundamental chemistry and solid state physics.

$\mathrm{CO}_{2}$ has a very complex phase diagram consisting of a number of crystalline molecular phases below $40 \mathrm{GPa}$. On further compression it polymerizes forming at moderate temperatures (up to $680 \mathrm{~K}$ ) amorphous glass with carbon in threefold and fourfold coordination [1], while the laser heating above $1800 \mathrm{~K} / 40 \mathrm{GPa}$ produces a polymeric covalent crystal phase $\left(\mathrm{CO}_{2}-\mathrm{V}\right.$, space group $I \overline{4} 2 d)$ that can be described as a network of fourfold coordinated carbon atoms interconnected by oxygen bridges resembling structurally $\beta$-cristobalite $\left(\mathrm{SiO}_{2}\right)[2]$.

The substantial kinetic barrier, reflecting dramatic changes in the bonding scheme on transition to the polymeric phase, led to numerous observations of metastable states in the stability field of $\mathrm{CO}_{2}-\mathrm{V}$, causing controversies. Hence, we have decided to investigate the chemical and phase stability of carbon dioxide at pressures up to $120 \mathrm{GPa}$ [3] and temperatures reaching $6000 \mathrm{~K}$ [4], an unexplored range in all the previous reports.

High-pressure high-temperature in situ X-ray diffraction patterns, here reported for the first time, proved that $\mathrm{CO}_{2}-\mathrm{V}$ is the only nonmolecular form of $\mathrm{CO}_{2}$ relevant to the Earth's deep interior. Moreover, contrary to the previous findings, no evidences for the decomposition of $\mathrm{CO}_{2}-\mathrm{V}$ into the elements have been found. Variation of the Bragg peak distribution on Debye-Scherrer rings at temperatures $>4000 \mathrm{~K}$ [4] may suggest a further possible extension of the stability field of this polymeric solid toward the pre-melting state. The presented findings play a pivotal role in understanding the behavior of hot dense carbon dioxide and provide a good basis for further experimental studies of $\mathrm{CO}_{2}$ at extreme pressures and temperatures.

[1] Santoro, M., Gorelli, F.A., Bini, R., Ruocco, R., Scandolo, S. \& Crichton, W.A. (2006). Nature 441, 857.

[2] Santoro, M., Gorelli, F.A., Bini, R., Haines, J., Cambon, O., Levelut, C., Montoya, J.A. \& Scandolo, S. (2012). Proc. Natl Acad. Sci. USA 109, 5176.

[3] Dziubek, K.F., Ende, M., Scelta, D., Bini, R., Mezouar, M., Garbarino, G. \& Miletich, R. (2018). Nat. Commun. $9,3148$.

[4] Scelta, D., Dziubek, K.F., Ende, M., Miletich, R., Mezouar, M., Garbarino, G. \& Bini, R. (2021). Phys. Rev. Lett. 126, 065701.

\section{Keywords: high pressure; carbon dioxide; deep Earth}

The authors thank the Deep Carbon Observatory initiative (Extreme Physics and Chemistry of Carbon: Forms, Transformations, and Movements in Planetary Interiors, from the Alfred P. Sloan Foundation) that supported this work and the ESRF for granting the beamtime. 